\title{
A multifunctional on-line brain stimulation system
}

\author{
RICHARD W. PHELPS \\ Tufts University, Medford, Massachusetts 02155 \\ and \\ MICHAEL J. LEWIS \\ Howard University, Washington, D. C. 20059
}

\begin{abstract}
A flexible on-line electrical brain stimulation system designed for brain stimulation reward (BSR) research is described. A Cromemco Z-2D microcomputer is interfaced with a constantcurrent stimulator and a standard operant chamber. The system programs, written substantially in BASIC, calculate BSR threshold by two rate-independent methods, measure rate of operant responding, and determine resistance of the brain. Other software programs are used for training rats on complex schedules of reinforcement, for system hardware calibration, and for sophisticated statistical data analyses.
\end{abstract}

Using a classical psychophysical technique to measure electrical brain stimulation reward (BSR) thresholds presents a significant problem. The subject's behavior (usually leverpressing) is controlled by the reinforcing stimulation for which the threshold is to be found. Many experimenters have tried to avoid this problem by measuring animal response rate using suprathreshold stimulation and inferring changes in threshold from changes in rate. If a rate measure is used, however, it is not possible to discriminate between the effects of the experimental treatment on threshold vs. its effects on motor responses.

Alternative methods (Huston \& Mills, 1971; Marcus \& Kornetsky, 1974; Valenstein \& Meyers, 1964) determine threshold with less reliance on rate of response. Huston and Mills (1971) measure BSR threshold with a psychophysical procedure based on the observation that performance under a fixed-ratio (FR) schedule is different from that under a continuous reinforcement (CRF) schedule (Ferster \& Skinner, 1957). In this procedure, rats leverpress for rewarding stimulation on an FR schedule and, concurrently, on a CRF schedule, using a single lever. This combined schedule is known as a CRF-FR. The FR current intensity is fixed at a suprathreshold level, which maintains the leverpressing response at any CRF current intensity.

The procedures developed in this paper are a direct outgrowth of the work of Drs. A. William Mills, M. P. Huston, and G. PickCassens and their associates. We wish to express our appreciation for their previous work and contribution to our thinking. The system development and purchase was partially supported by grants from Bernard Harleston, Dean of Faculty, Tufts University; DHHS (RR 07179, RR 08016); and Howard University, Graduate School of Arts and Sciences (NIH-RPE 1397F). Reprint requests should be addressed to Michael J. Lewis, Department of Psychology, Howard University, Washington, D. C. 20059.
An animal performing on a "pure" FR schedule exhibits postreinforcement pauses (PRPs) (Ferster \& Skinner, 1957). As CRF current intensity is increased from zero on a CRF-FR schedule, FR pauses become shorter and eventually disappear. The rat's performance shifts from that which is characteristic of an FR schedule (many PRPs) to that which is characteristic of a CRF schedule (no PRPs). Decreasing the CRF current intensity causes the pauses to reappear. Threshold is determined by appearance or disappearance of these pauses as the CRF current intensity is varied. Huston and Mills (1971) reported that threshold determination was independent of the size of the FR and of the supratheshold FR current intensity.

The definition of a PRP has been a problem using this threshold technique. Huston (Note 1) defined a PRP as the interval just visually discernible on the cumulative recorder. Cassens and Mills (1973) defined it as an interval greater than $7 \mathrm{sec}$, but not more that $3 \mathrm{~min}$. Cassens, Shaw, Dudding, and Mills (1975) devised a ratedependent definition: A PRP was defined as an interval greater than the mean CRF interresponse interval (IRI) plus three standard deviations. Thus, a PRP was relative to the CRF IRI. This provided a rate-independent means of determining the PRP and, hence, threshold.

The system presented in this paper employs the same rate-independent concept for determining threshold. A fixed number of FR reinforcements are presented at each CRF current level. Threshold is defined as the current level that produces PRPs half of the time, that is, a PRP/FR ratio of .50 . Threshold is determined by evaluating PRP/FR ratios over a CRF current range, and then interpolating the current value at a PRP/FR of .50 (see Figure 1).

Threshold determination using this system is reliable 


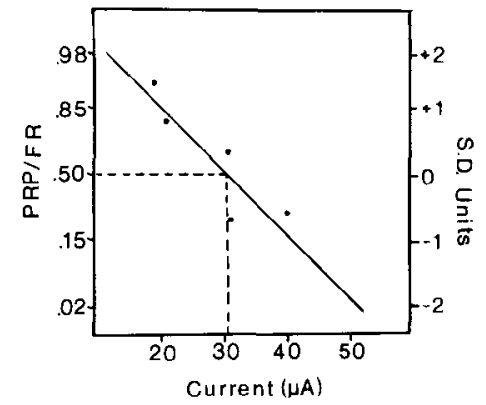

Figure 1. Postreinforcemenit pauses per fixed-ratio reinforcement (PRP/FR) as a function of current intensity (from Cassen, 1975).

and independent of an animal's rate of response. This is essential for many physiological and psychopharmacological BSR experiments. One drawback to previous threshold determinations (Cassens et al., 1975) using this method is the requirement of approximately $2 \mathrm{~h}$ for a single BSR session (Cassens, Note 2). Another drawback is that occasionally threshold cannot be determined because of either floor or ceiling effects with the PRP/FR ratio. The PRP/FR ratio can be 0 or 1.0 for all CRF current levels; thus, no threshold can be calculated (Huston, Note 1). To avoid this problem an alternative method of threshold determination, the "z-pause," has been developed. With this method it is assumed that with virtually every FR there is a PRP of some duration. The mean PRP interval at each CRF current level is compared with the mean IRI (i.e., during CRF responding). This also results in a rate-independent measure of threshold. No floor or ceiling effects result and, thus, threshold can always be determined.

The principal reason for the development of this system is the need for a reliable, more flexible, and inexpensive computerized BSR threshold system that permits faster threshold determination over a variety of experimental situations (Phelps \& Lewis, Note 3 ). The use of the microcomputer-based system detailed here accomplishes these goals.

\section{CONST ANT-CURRENT STIMULATOR}

Constant electrical current is required for most BSR threshold methods. Wayner, Peterson, and Florczyk (1972) and Emde and Shipton (1970) described such stimulators. Mills and his collaborators (Cassens et al., 1975) designed a constant-current stimulator based on a modification of a system described by Wayner et al. (1972). Constant current is maintained by voltage vary. ing as a function of brain resistance in the circuit. Voltage is monitored by the use of a FET instrumentation amplifier in the stimulator (Cassens et al., 1975). The stimulator to be described is of this basic design, but modified slightly for better current regulation over a broader resistance range. [Current regulation was empirically determined through extensive testing of the design over several years (Phelps \& Lewis, Note 3).] The components of this stimulator are provided in Table 1 .

The operation of the stimulator may be described in five stages (see Figure 2). In Stage 1, the 8-bit digital output (DO) from the microcomputer, which sets the stimulator current level, is buffered through two quad bistable latches (IC). This provides for bit stabilization. The digital signal is then passed to the $\mathrm{D} / \mathrm{A}$ converter (Stage 2) and converted to an analog voltage. The ana$\log$ output $(0-1 \mathrm{~V})$ is negatively offset with respect to ground and amplified with an operational (OP) amplifier (A1), forming a symme tric biphasic output (Stage 3). $\mathrm{R} 1$ is trimmed to adjust the amplification of Stage 3. Stage 4 is based on the Howland current pump and holds the output current constant by varying the voltage as a function of brain resistance via a feedback loop (R6) in the $\mathrm{OP}$ amplifier circuit. The constant-current regulation across a wide resistance range (see Figure 4) is trimmed with R8. Currents from 1-127 microA can be delivered

Table 1

List of Components for Computer-Operated Constant-Current Stimulator

\begin{tabular}{|c|c|c|}
\hline Quantity & Label & Description \\
\hline 2 & IC & Texas Instruments quad bistable latch SN7475N \\
\hline 1 & $\mathrm{D} / \mathrm{A}$ & Hybrid Systems D/A converter 371.8 \\
\hline 2 & A1 & Teledyne Philbrick 1005 operational amplifier \\
\hline 1 & A2 & Teledyne Philbrick 4253 FET instrumentation amplifier \\
\hline 1 & \pm 15 & Teledyne Philbrick 2203 dc output regulated power supply \\
\hline 1 & R1 & $2-\mathrm{KB}$ rheostat \\
\hline 1 & R2 & $510 \mathrm{~W} 2 \% .25 \mathrm{~W}$ \\
\hline 1 & R3 & $12 \mathrm{~KB} 2 \% .25 \mathrm{~W}$ \\
\hline 2 & R4 & 50-KB potentiometers \\
\hline 2 & R5 & $20 \mathrm{~KB} 2 \% .25 \mathrm{~W}$ \\
\hline 2 & R6 & $40 \mathrm{~KB} 2 \% .25 \mathrm{~W}$ \\
\hline 1 & R7 & $50 \mathrm{~KB} 2 \% .25 \mathrm{~W}$ \\
\hline 1 & R8 & 50-KB rheostat \\
\hline 1 & R9 & $2 \mathrm{~KB} 2 \% .25 \mathrm{~W}$ \\
\hline 1 & R10 & 10-KB potentiometer \\
\hline 1 & R11 & $80.6-\mathrm{KB} 1 \% .25 \mathrm{~W}$ \\
\hline 2 & R12 & $1002 \% .25 \mathrm{~W}$ \\
\hline 1 & $\mathrm{C} 1$ & $50 \mathrm{microF}$ \\
\hline 2 & $\mathrm{C} 2$ & $.001 \mathrm{micr}$ oF \\
\hline
\end{tabular}




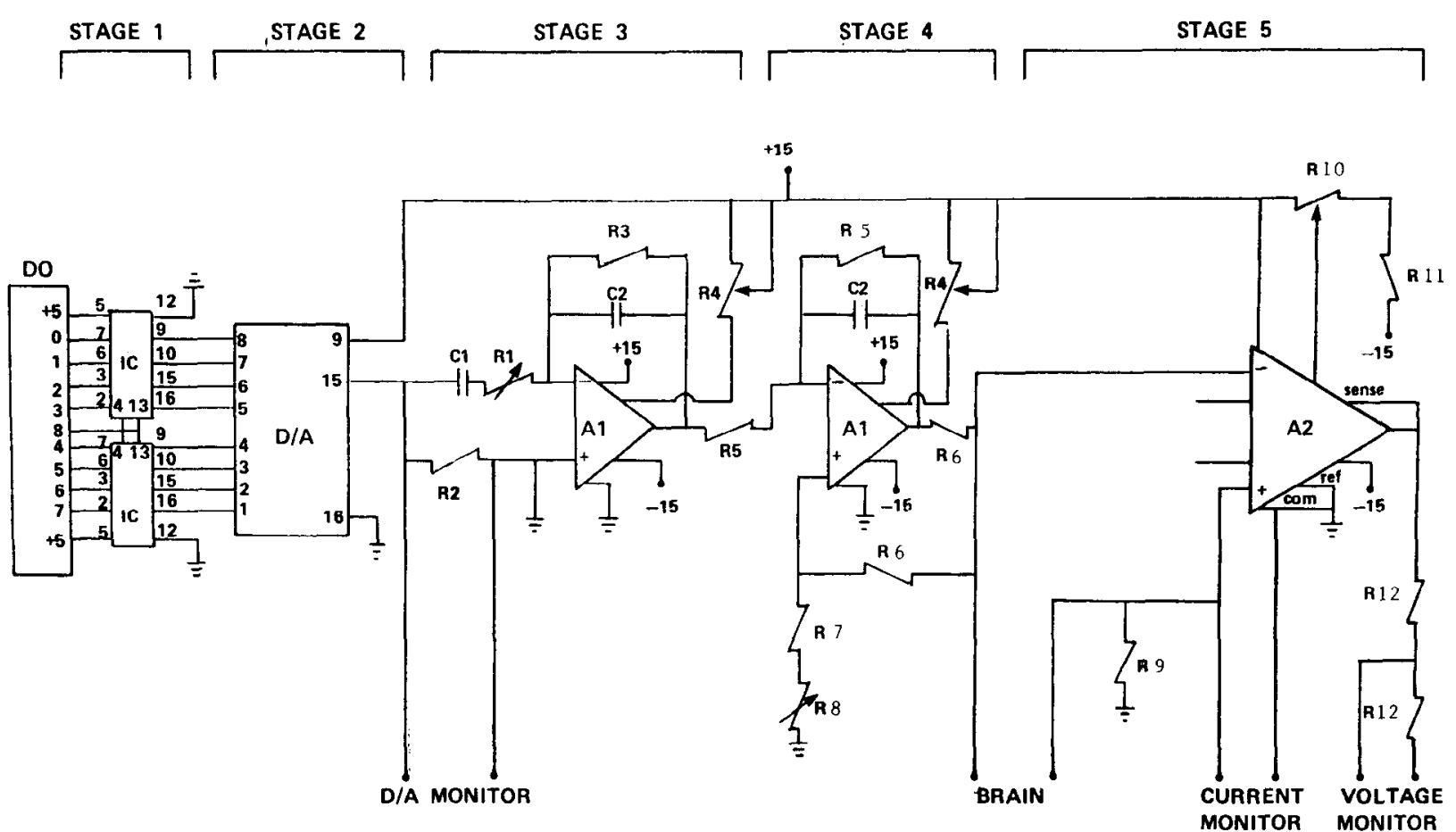

Figure 2. Schematic of compu ter-operated constant-current stimulator (see text for symbols and discussion).

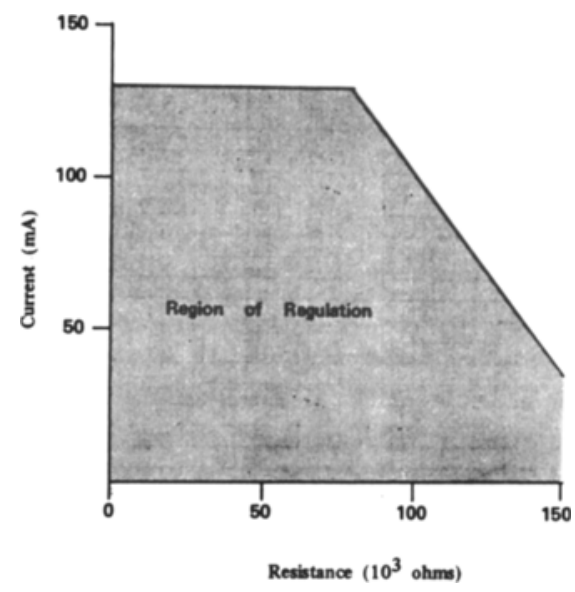

Figure 3. Region of stimulator constant-current regulation.

with 1 -microA resolution. The region of regulation of the stimulator is shown in Figure 3.

Stage 5 utilizes a FET amplifier with unity gain. The FET input is in parallel with the electrode tips and the output returns stimulator voltage to the $A / D$ converter of the computer. This voltage information is utilized for on-line resistance calculations. The FET amplifier has the principal advantage of a high input and low output impedance, and thus does not interfere with voltage measures across the electrode tips or the computer $\mathrm{A} / \mathrm{D}$ operation.

Zero trims for each stage of the stimulator circuit are provided by potentiometers R4 and R11. Both R12s serve as a voltage divider on the output of the FET ampli- fier, and scale the voltage from a \pm 10.00 - $V$ maximum to $\pm 5.00 \mathrm{~V}$. This voltage is more compatible with the $\pm 2.50-\mathrm{V}$ input range of the computer's $\mathrm{A} / \mathrm{D}$ converter. R9 provides for current monitoring and serves to isolate animal electrode tips from ground.

\section{HARDWARE}

The microcomputer is a Cromemco Model Z-2D with $48 \mathrm{~KB}$ of random access memory (RAM). Computer peripherals include two 5-in. floppy disk drives, a D+7A I/O board with multichannel analog and digital $\mathrm{I} / \mathrm{O}$, and a Teletype 43 (TTY43).

\section{SOFTWARE}

The software is written in Cromemco 16-KB disk extended BASIC and Z80 assembler. Programs for equipment calibration, schedule presentation, data storage, and analysis are all in BASIC.Cross-compatible modularization between programming languages provides the ease and flexibility of BASIC programming while meeting the real-time demands of the stimulator operation and current monitoring with an assembly language program. Thus, modification of the experimental paradigm or schedule parameters is easily accomplished as changes in experiment procedures are indicated.

The computer software package consists of seven modules. (1) MENU executes computer initialization and provides branching to the other BASIC programs within the system. (2) TRAIN permits CRF and concurrent CRF/FR training of rats. (3) PRESS detects lever- 
presses and times IRIs. (4) STIM operates the constantcurrent stimulator and monitors output voltage. (5) EBS handles the formatting of the complex stimulus presentation and on-line storage of data collection. (6) STAT performs all statistical analysis after data acquisition has been completed. (7) CAL calibrates stimulator operations. MENU, EBS, STAT, and CAL are BASIC programs; PRESS and STIM are written in assembly language.

The operation of these programs requires almost all $48 \mathrm{~K}$ of RAM for storage of the operating system, the BASIC interpreter, program, and temporary data storage. The flowchart of the EBS program operation is shown in Figure 4 and described below. First, the experimenter is prompted to enter the complex schedule parameters at the TTY43 terminal (see Figure 5). Then a sequence of system diagnostics is executed before each threshold determination. The experimenter is instructed to insert a known resistive load $(51 \mathrm{kohms})$ into the stimulator circuit for system calibration. Once the resistor is in place, the user presses RETURN to initialize the calibration routine. The program moves the stimulator through a current range of 1.127 microA in 1-microA steps. Based on the voltage across the standard resistor returning through the computer's A/D converter, a multiplication factor $(M)$ is calculated. This factor, when multiplied times A/D values (A), produces a measure of resistance (R), accurate to $\pm 1.5 \mathrm{kohms,}$, as presented: $\mathrm{R}=(\mathrm{A} \times \mathrm{M}) / \mathrm{l}$. The multiplication factor is reported to the user and if it does not lie in a tolerance range of \pm 2 , the constant-current stimulator calibration should be checked. The maximum positive and negative deviation in calculated resistance from the 51-KB standard over the

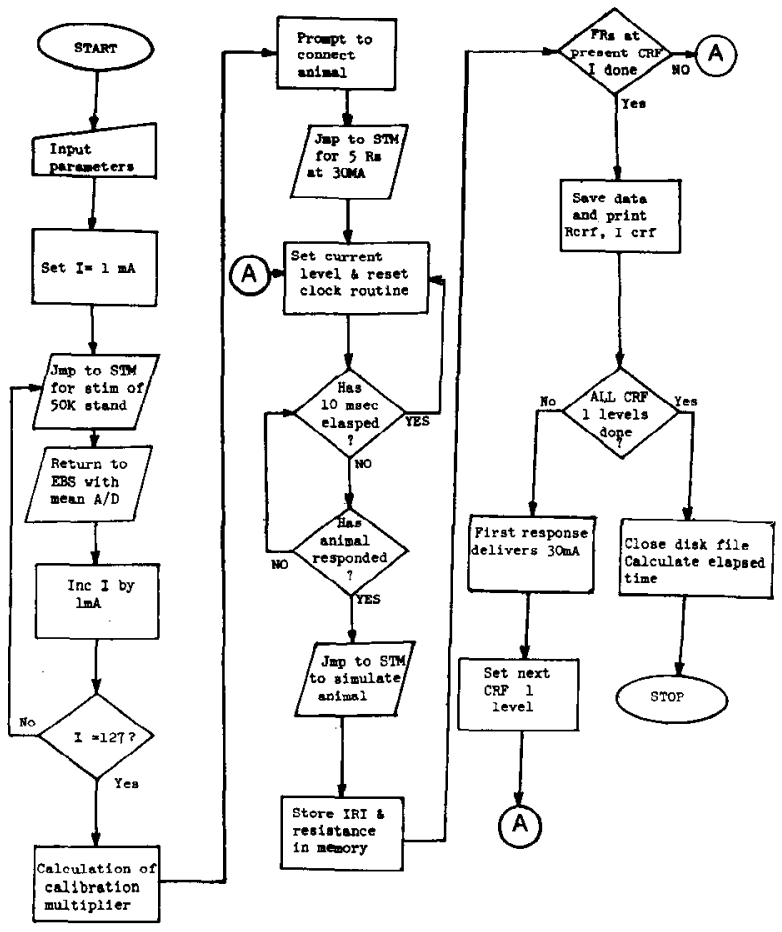

Figure 4. Flowchart of the EBS program (see text).

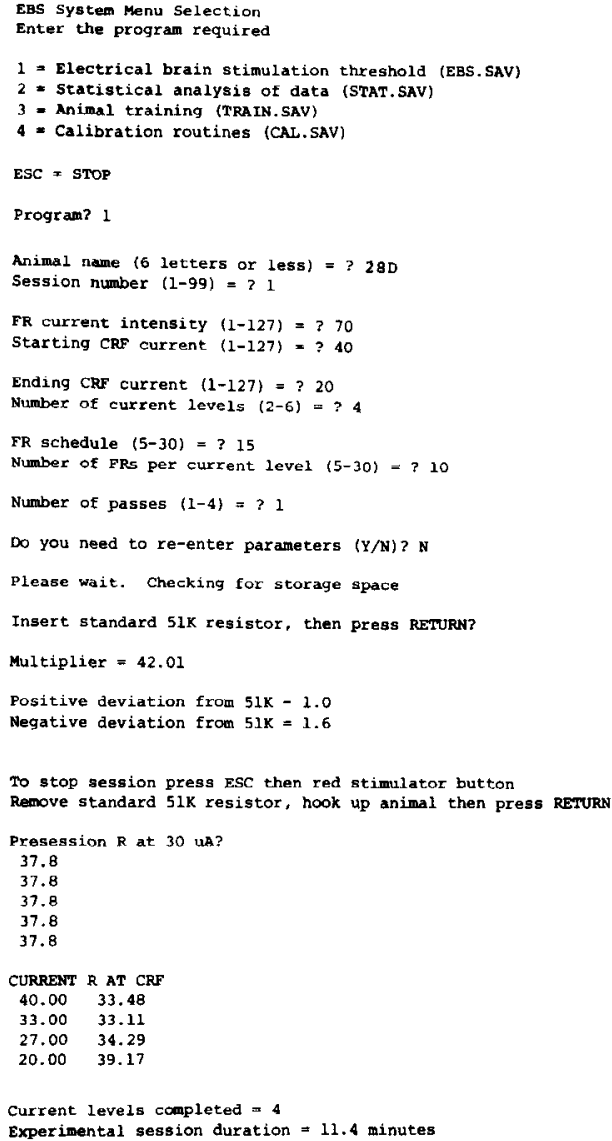

Figure 5. Example of an EBS program printout.

127-microA range is also reported. Once the calibration is complete, the experimenter is reminded to remove the standard resistor and then connect the electrode cable to the animal. When the animal is connected and in the testing cage, RETURN is pressed and the program proceeds to deliver five consecutive stimulations at 30 microA, spaced 1 sec apart. These stimulations provide a measure of baseline resistance at a value common to all the animals.

The program then waits in a loop for a lever response. Each leverpress causes closure between ground and Pin 0 of the computer digital input bits. The first response at each CRF current level results in stimulation at the standard 30 microA. This is done only on the first response and provides a standard resistance measure at each current level during threshold determination. While waiting for each response, the PRESS program cycles through a clock routine to count elapsed time in units of $10 \mathrm{msec}$. When no other tasks, such as stimulator operations, are active, the program waits in this central loop measuring the IRI. A maximum of $5.5 \mathrm{~min}$ is allowed to elapse before one stimulation is automatically delivered and the clock is reset. Once a response is made, the PRESS program stops timing and control is passed to the EBS program where the IRI value is stored. Then STIM is called with the value of the next stimulation current intensity. The stimulus is delivered and 
mean returning voltage is calculated. Control retums to the EBS program. The brain resistance is then calculated based on the mean voltage stored along with the IRI in RAM. The EBS program checks whe ther the CRF current level is finished and returns to the PRESS program. If the level is not finished, the program waits for another response in PRESS. If the CRF level is finished, the IRIs and resistances for all responses at that current level are stored on floppy disk drive B. The completed CRF current level and mean CRF resistance for that current level are reported on the TTY43. The program then checks whether all CRF current levels are complete. If all levels are not finished, the next CRF current value is set and the program waits for another response in PRESS. If all measures have been taken, the program closes the data file and prints the total elapsed time. The program then returns to MENU.

At any point during the execution of EBS, the program may be aborted by typing ESC; all collected data are retained for partial analysis. This feature avoids the loss of data if an animal does not complete the session.

The STIM module is called forth from Programs EBS, CAL, and TRAIN. STIM is responsible for the real-time generation of the stimulation waveform, and it rectifies and averages returning stimulator voltage and compensates for any dc offset in the signal. Retuming voltage is sampled at 38-KB samples/sec. A flowchart of STIM is shown in Figure 6. STIM generates either mono- or

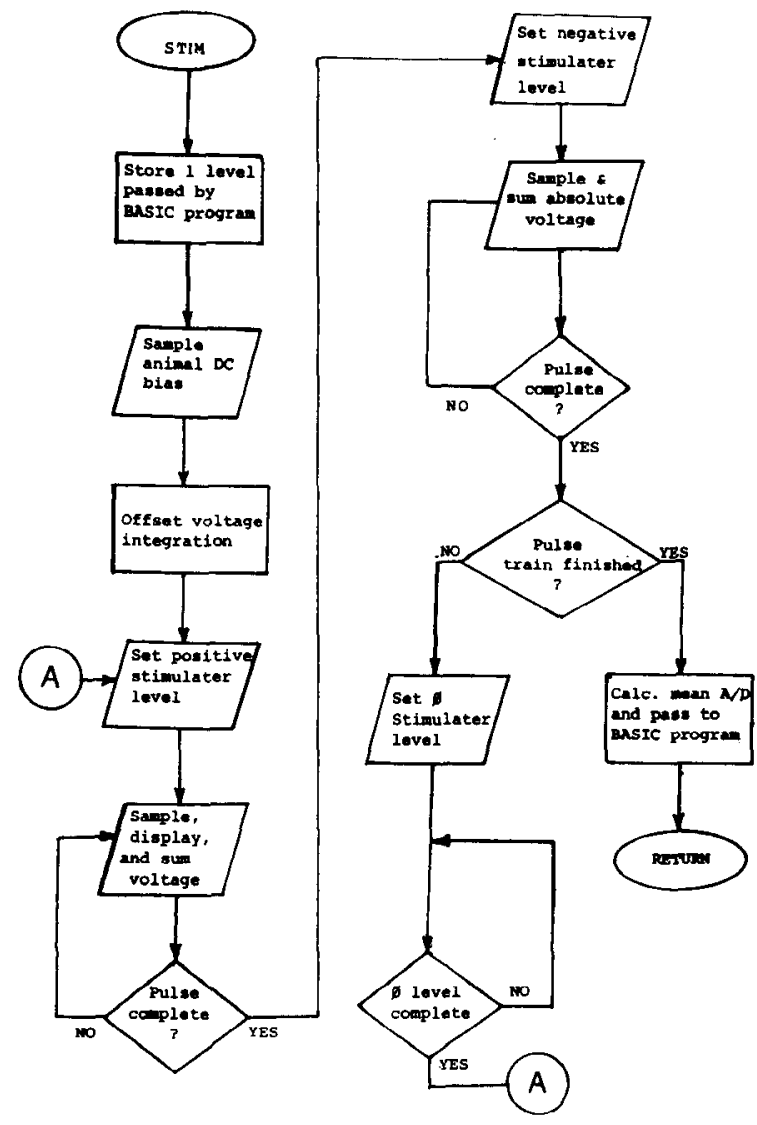

Figure 6. Flowchart of the STIM program.

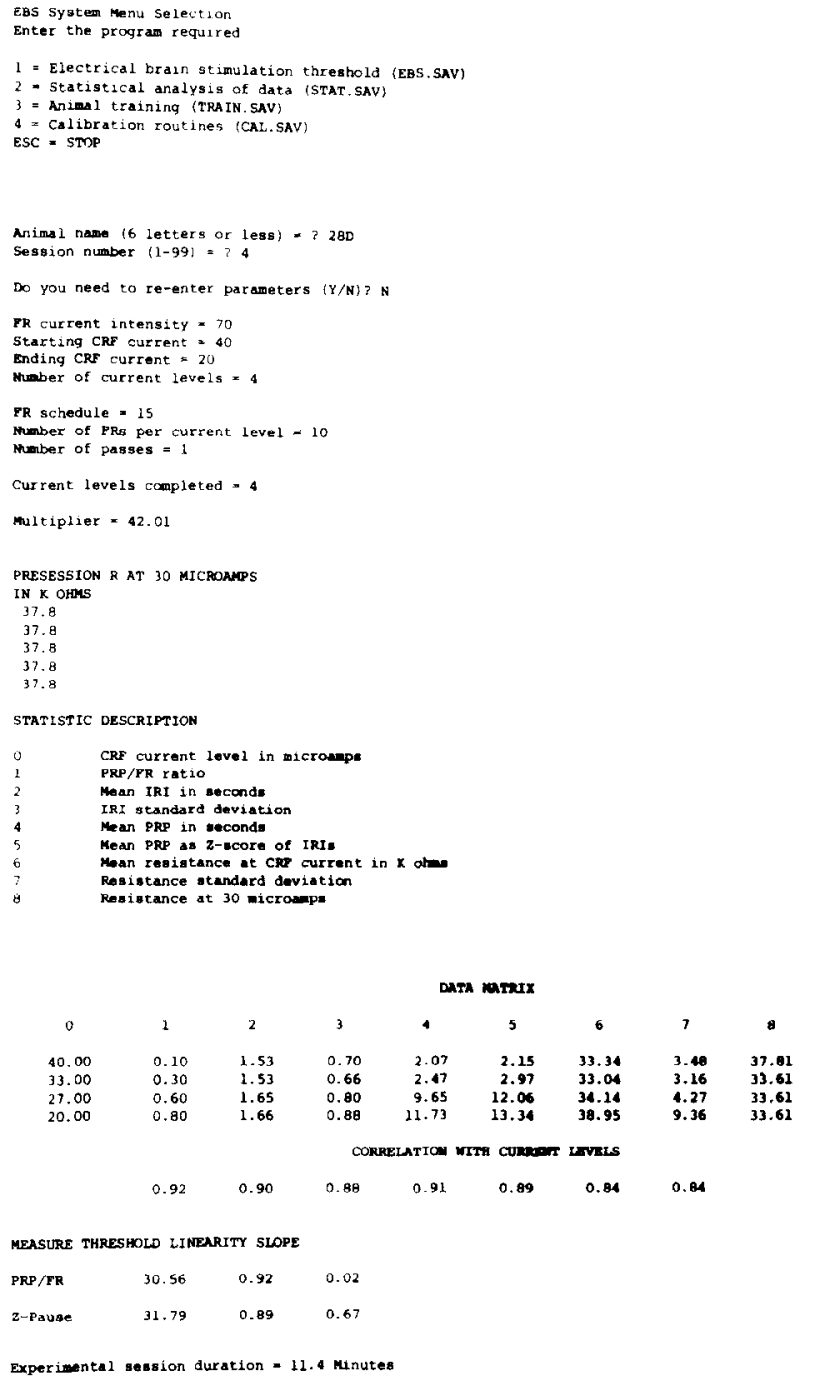

Figure 7. Example of a STAT program printout.

biphasic pulses of variable duration and frequency. Pulse phase, duration, frequency, and train length are each set by modifying a single instruction of the STIM program.

CAL is used to calibrate stimulator current levels, current regulation, stimulus, waveform, and computer A/D.

The STAT program provides a comprehensive statistical analysis of each session, including two alternate calculations of the threshold. STAT utilizes the IRI and resistance data stored on the floppy disk by EBS. The experimenter first inputs the animal's name and the session number so that the data may be located on the floppy disk (see Figure 7). A summary of the CRF/FR schedule parameters and the multiplication factor (M) is printed. After the multiplication factor $(\mathbf{M})$ is printed, a table of the various types of statistical measurements and their abbreviations is printed. This is followed by printing resistance values (in kilohms) of the five initial stimulations of each rat at the standard (across all animals) 30-microA current. This permits determination 
of brain resistance of each animal at the beginning of each BSR session. The data are then read from the floppy disk one current level at a time. A data matrix is then reported that includes the following measures of each CRF current level: (1) the PRP/FR ratio, (2) the mean IRI in seconds, (3) the IRI standard deviation, (4) the mean PRP duration for all FRs at each current level in seconds, (5) the mean PRP duration expressed as the number of standard deviations from the mean IRI, (6) the mean resistance of the CRF current intensity in kilohms, (7) the standard duration of the CRF resistance, and (8) the resistance values in kilohms of a 30 -microA stimulation at the beginning of each CRF current level (for evaluation of brain resistance throughout the BSR session).

A correlation matrix (using Pearson's r) of CRF current levels with Measures 1 through 7 is then printed. BSR threshold is then calculated based upon the PRP/ FR ratio method described above. In addition, threshold is determined by the second method, "Z-pause." Z-pause threshold is the CRF current level that results in a mean PRP 2 standard deviations greater than the mean CRF IRI. This measure is an altemative way of calculating BSR threshold. With both methods, the equation of the best-fitting line for CRF current values vs. the PRP measurement at each CRF current is calculated using a least squares regression analysis. Threshold is determined by interpolating the CRF current that corresponds with a PRP/FR ratio of .50 or a z-pause of 2 .

The final datum printed is the duration in minutes of the BSR session. The program then returns to the MENU program, ready for another operant session, training, or program.

\section{SUMMARY}

This system has been used to de termine BSR threshold, rate of response, and brain resistance in various brain sites, including the lateral hypothalamus, medial forebrain bundle, midbrain ventral noradrenergic bundle, and locus coeruleus. Investigations of aging processes, effects of heat and cold stress, and the effects of several types of drugs have been accomplished.

The length of time to complete an operant session is variable depending upon: (1) the rate of leverpressing by the animal, (2) the number of CRF current intensities tested, and (3) the need to descend or to both descend and ascend the CRF intensity range. We typically use four CRF intensities, as indicated in Figure 5. Animals with implants in a number of positive sites (e.g., lateral
Table 2

Approximate Cost of the Brain Stimulation System

\begin{tabular}{lr}
\hline \multicolumn{1}{c}{ Component } & \multicolumn{1}{c}{ Cost } \\
\hline Cromemco System 2 Mìcrocomputer & $\$ 4,700$ \\
D+7A I/O Multichannel Interface & 295 \\
Teletype 43 & 1,350 \\
Stimulator Assembly & 400 \\
Stimulator Assembly & 600 \\
Software & 2,000 \\
Total & $\$ 9,345$ \\
\hline
\end{tabular}

hypothalamus) complete a session in approximately $15 \mathrm{~min}$.

Table 2 provides the approximate cost of this system and its major components based on currently available prices.

\section{REFERENCE NOTES}

1. Huston, J. Psychophysics or energizing and reinforcing stimulation of the brain. Unpublished dissertation, Tufts University, 1969.

2. Cassens, G. Electrical self-stimulation in the ventral noradrenergic bundle of the rat. Unpublished dissertation, Tufts University, 1975.

3. Phelps, R., Lewis, M. J. Stability in threshold and impedance parameters of brain stimulation reward. Manuscript in preparation, 1982.

\section{REFERENCES}

Cassens, G., Mills, A. Lithium and amphetamine: Opposite effects on threshold of intracranial reinforcement. Psychopharmacologia, 1973, 30, 283-290.

Cassens, G., Shaw, C., Dudding, K., \& Mills, A. On-line brain stimulation: Measurement of threshold of reinforcement. Behavior Research Methods \& Instrumentation, 1975, 7, 145150.

Emde, J. W., \& Shrpron, H. W. A digital controlled constant current stimulator. Electroencephalography and Clinical Neurophysiology, 1970, 29, 310-313.

Fensten, M., \& Sxinnen, B. Schedule of reinforcement. New York: Appleton-Century-Croft, 1957.

Huston, J., \& Mills, A. Threshold of reinforcing brain stimulation. Communications in Behavioral Biology, 1971, 5, 331340.

Marcus, R., \& Konnetsry, C. Negative and positive intercranial reinforcement thresholds: Effects of morphine. Psychopharmacologia, 1974, 38, 1-13.

Valengtein, E., \& MrYers, W. Rate independent test of reinforcing consequences of brain stimulation. Journal of Comparative and Physiological Psychology, 1964, 57, 52-60.

Wayner, M. J., Petenenon, R., \& Florczyk, A. A constant device for brain stimulation. Physiology \& Behavior, 1972, 8, 1189-1191.

(Received for publication May 25, 1981; reviaion accepted March 25, 1982.) 